seven consecutive days electronically. On Day 7, participants completed three cognitive tasks (Ravens Standard Progressive Matrices, Wisconsin Card Sorting task and a Mental Rotation task). Standard measures of sleep continuity were derived from sleep diaries, including sleep onset latency (SOL), number of awakenings (NWAK), wake after sleep onset (WASO), time in bed (TIB) and sleep efficiency (SE\%). Three Pearson's correlations were conducted between the cognitive tasks and sleep continuity measures. All p-values were adjusted for multiple comparisons (adjusted p-value $=.01$ ).

Results Overall, no association was found between subjective sleep continuity (SOL, NWAK, WASO, TIB and SE\%) and Ravens Standard Progressive Matrices, Wisconsin Card Sorting task, or Mental Rotation accuracy scores (all p-values > $.01)$.

Discussion These results indicate that fluid intelligence, mental rotation and set-shifting are not associated with subjective sleep continuity. Previous literature has found associations between objective sleep measures and sleep spindle activity (Bódizs et al., 2014; Ujma et al., 2015), therefore fluid intelligence may only be associated with objective sleep architecture.

\section{CHARACTERISTICS OF INITIAL SLEEP STUDY IN INFANTS WITH PRADER WILLI SYNDROME}

${ }^{1}$ Laura Gardener*, ${ }^{1} \mathrm{~N}$ Ghosh, ${ }^{1} \mathrm{R}$ Pabary, ${ }^{2} \mathrm{~N}$ Bridges, ${ }^{1} \mathrm{~F}$ Trucco, ${ }^{1} \mathrm{H}$ Tan, ${ }^{1}$ Ruth O'Reilly. ${ }^{1}$ Royal Brompton Hospital, UK; ${ }^{2}$ Chelsea and Westminster Hospital

10.1136/bmjresp-2021-bssconf.42

Introduction Patients with Prader Willi syndrome are predisposed to problems with ventilatory control and sleep disordered breathing. This may be further impacted by the commencement of growth hormone in infancy. As a result early monitoring with sleep study is indicated, however it can be challenging to effectively council families about the expected results in the first few months of life due to the paucity of data pertaining to infants reported in the literature. Methods We retrospectively collected first sleep study and demographic data from 36 children with a confirmed diagnosis of Prader Willi syndrome as determined by tertiary endocrine assessment and genetic variant.

Abstract 48 Table 1 First sleep study parameters of infants with Prader Willi Syndrome. Results are reported in medians with interquartile ranges. $(\mathrm{TCPCO} 2=$ transcutaneous carbon dioxide, $\mathrm{AHI}=$ apnoea hypopnoea index)

\begin{tabular}{|l|c|}
\hline \multicolumn{1}{|c|}{ Characteristic, $\mathbf{n = 3 6}$} & Result \\
\hline Gender M:F (\%) & $23(64 \%): 13(36 \%)$ \\
\hline Age at first study, Decimal years & $0.57(0.46,0.75)$ \\
\hline ODI 3\%, events per hr (n= 33) & $7.44(4.3,12.5)$ \\
\hline Mean Oxygen Saturations, \% & $97.2(96.1,98.6)$ \\
\hline Minimum Oxygen Saturation, \% & $86(81,88.8)$ \\
\hline Mean TcPCO2, kPa ( $\mathbf{n = 3 5 )}$ & $5.5(5.15,5.8)$ \\
\hline Maximum TcPCO2, kPa ( $\mathbf{n}=\mathbf{3 5})$ & $6.1(5.7,6.5)$ \\
\hline Total AHI, events/hour (n= 27) & $3.8(1.9,9.1)$ \\
\hline Obstructive AHI (OAHI), events/hour $(\mathbf{n}=\mathbf{2 6})$ & $0.95(0.1,2.8)$ \\
\hline Central AHI (CAHI), events/hour $(\mathbf{n}=\mathbf{2 6})$ & $1.65(0.3,4.8)$ \\
\hline Mixed AHI (MAHI), events/hour $(\mathbf{n}=\mathbf{2 6})$ & $0(0,0.13)$ \\
\hline Undefined AHI (UAHI), events/hour $(\mathbf{n}=\mathbf{2 6})$ & $0.00(0.00,0.1)$ \\
\hline
\end{tabular}

Results The clinical characteristics and sleep study parameters are described in table 1 . Whilst the median results related to both gas exchange and ventilation are reassuring, 6 (23\%) patients of the 26 who had a breakdown of their AHI had an OAHI $>5$, with a maximum recorded OAHI of 27.7. Six (23\%) of the 26 who had a breakdown of their AHI had a CAHI $>5$, with a maximum recorded CAHI of 9.9

Discussion This data shows that whilst the majority of patients who undergo baseline sleep study for Prader Willi syndrome will have a normal result, $23 \%$ may be expected to have an excess of obstructive events. This project may serve as useful pilot data to help council families ahead of the initial sleep study. It is our intention to follow these patients and collect longitudinal data to determine if baseline results are predictive of future need for respiratory support or response to growth hormone.

\section{A PILOT META-ANALYSIS ON CONCUSSION-RELATED SLEEP DISTURBANCES}

1,2Suleyman Noordeen, 1,3 Mehmet Ergisi, 1,2Rebeka Popovic, 1,3Abinayan Mahendran 1,3 Mohammed Memon, ${ }^{1,3}$ Saahil Hegde, ${ }^{1,3}$ Poh Wang, ${ }^{1,3}$ Connor Qiu, 1,2 Yizhou Yu* ${ }^{1}$ International Sleep Charity, UK; ${ }^{2}$ University of Cambridge, UK; ${ }^{3}$ Imperial College London, UK

10.1136/bmjresp-2021-bssconf.43

Fatigue and poor sleep have been shown as factors of postconcussive symptoms (Broshek et al., 2015, Brain injury). Students, previously reported to have poor sleep, thus might be at increased risk of concussion-related sleep disturbances (McGrath et al., 2010, Journal of athletic training). This study explored the relationship between concussion and sleep, to serve as a pilot for a systematic meta-analysis.

We searched the following databases MEDLINE, EMBASE, CINAHL, Web of Science, PsycINFO, Cochrane Central Register of Controlled Trials, MedNar and OAIster with the search terms: sleep, concussion, mild traumatic brain injury, college/ university and sports. Only studies using the Epworth sleepiness scale (ESS) and the Pittsburgh sleep quality index (PSQI) were included. Studies were excluded if they included nonhuman animals, patients with other neurological conditions or military personnel. Two researchers performed the filtering independently.

Our search produced 4460 results. We removed 834 duplicates and examined a random subset of 8 studies that met our exclusion criteria as a pilot study. Our analysis totaled 1086 participants (683 concussed). Using a randomeffects model containing 4 studies per outcome test, we found that concussion was associated with increased PSQI (standardised mean difference: $0.76,95 \%$ confidence intervals $[0.52,1.01]$, ?2: 0.03, I2: 49.28\%, p value < 0.0001), and ESS (standardised mean difference: $0.47,95 \%$ confidence intervals $[0.24,0.70], ? 2: 0.02, \mathrm{I} 2: 42.56 \%, \mathrm{p}$ value $<0.0001)$.

Our results suggest a diagnosis of concussion or mild traumatic brain injury is associated with lower sleep quality, as measured by PSQI, and sleepiness as measured by ESS, in this population. Further analyses of the rest of our dataset, covariates of the studies, verifications of the updated literature and assessments of the quality of each study are required. 